\title{
Maximum Acceptable Vibrato Excursion as a Function of Vibrato Rate in Musicians and Non-musicians
}

Vatti, Marianna; Santurette, Sébastien; Pontoppidan, Niels H.; Dau, Torsten

Publication date:

2013

Document Version

Publisher's PDF, also known as Version of record

Link back to DTU Orbit

Citation (APA):

Vatti, M., Santurette, S., Pontoppidan, N. H., \& Dau, T. (2013). Maximum Acceptable Vibrato Excursion as a Function of Vibrato Rate in Musicians and Non-musicians. Poster session presented at 166th Meeting of the Acoustical Society of America, San Francisco, California, United States.

\section{General rights}

Copyright and moral rights for the publications made accessible in the public portal are retained by the authors and/or other copyright owners and it is a condition of accessing publications that users recognise and abide by the legal requirements associated with these rights.

- Users may download and print one copy of any publication from the public portal for the purpose of private study or research.

- You may not further distribute the material or use it for any profit-making activity or commercial gain

- You may freely distribute the URL identifying the publication in the public portal 
Maximum Acceptable

Vibrato Excursion as a Function of Vibrato Rate in Musicians and Non-musicians
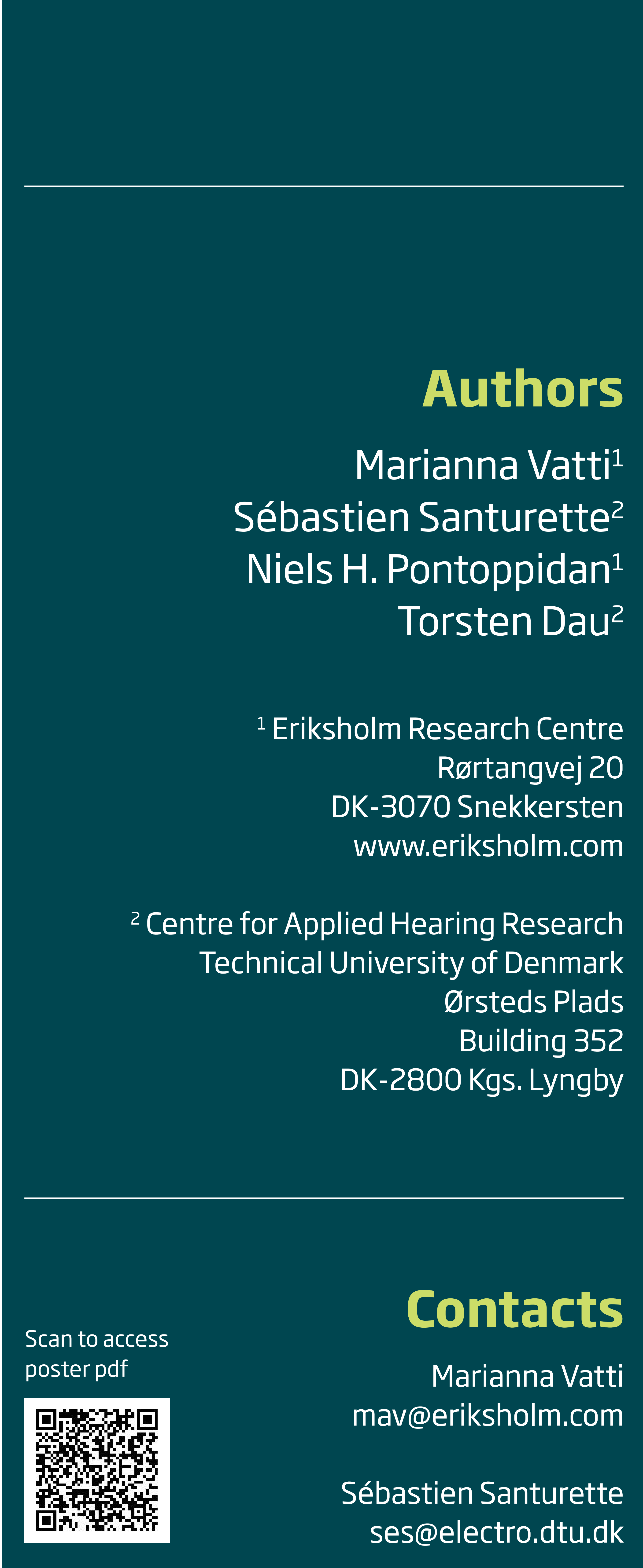

This study was concerned with the way in which the maximum acceptable vibrato excursion varies as a function of vibrato rate in normal-hearing $(\mathrm{NH})$ musicians and non-musicians.

\section{Background and aim of the study}

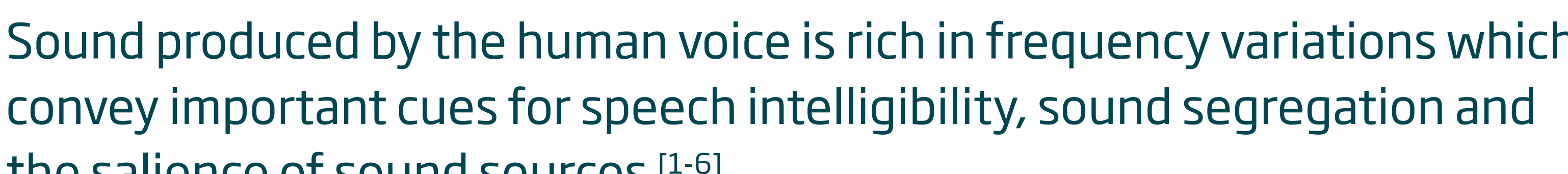
This study focused on the frequency variations that occur in the vocal vibrato of human singing voices. Vocal vibrato is physically described
as coherent fluctuations imposed on the harmonic components of

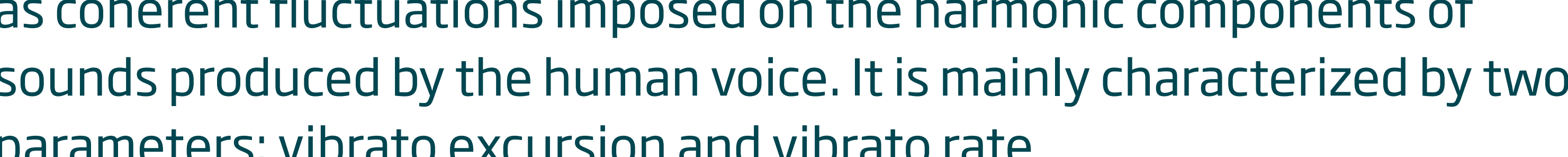
These parameters have been found to exhibit an interaction both in
physical recordings of singers' voices physical reecto
ratings ${ }^{100}$.
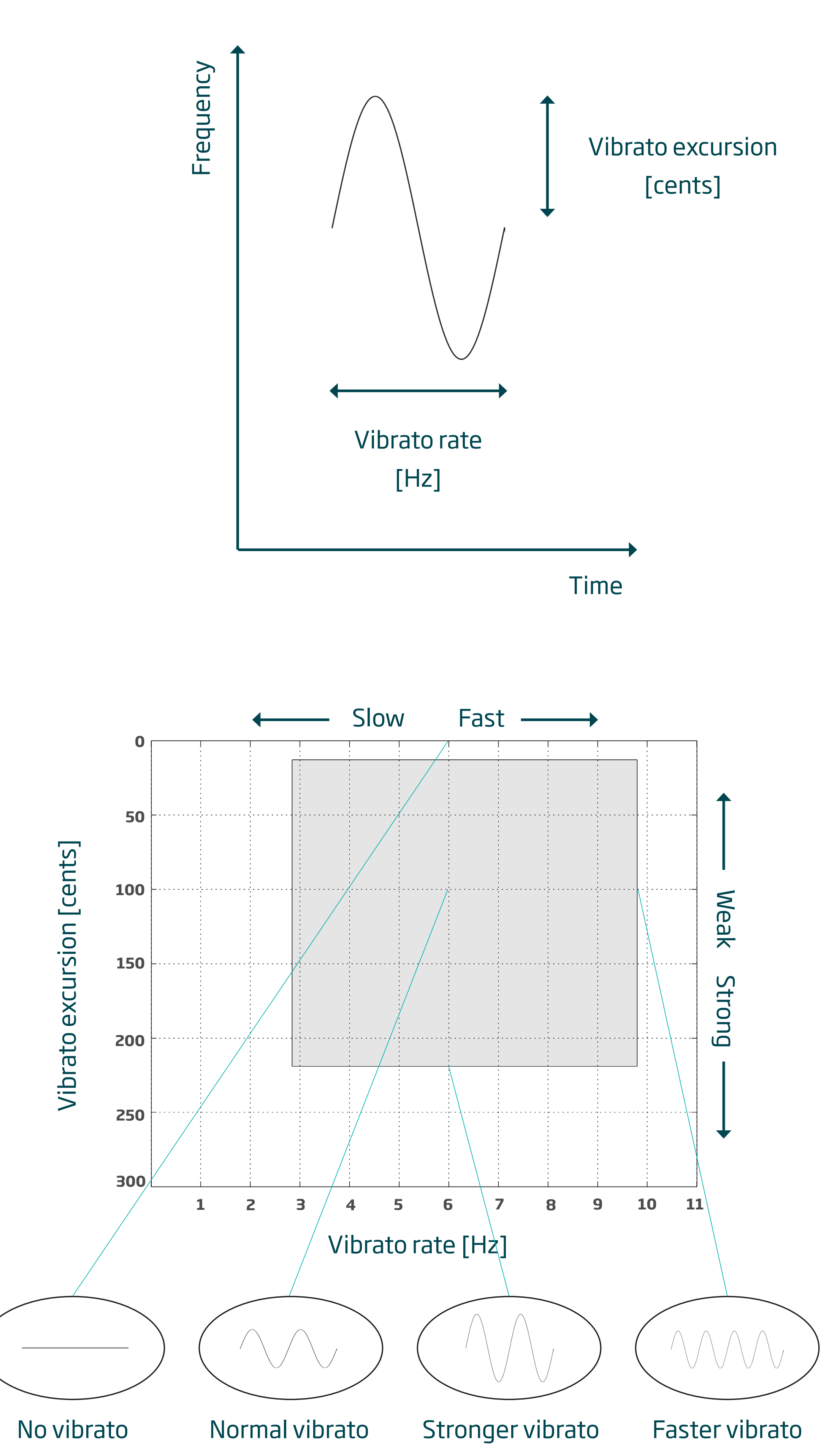

\section{Research questions}

sion vary across vibrato rate? 2) If so, how does this interaction between vibrato rate and excursion
compare with the maximum vibrato excursions produced by singers? 3) Does musical training affect the preference of the listeners

\section{Methods}

Stimulus configuration

- Coherent fluctuations applied by

adding the same frequency shift $M \bullet$ Three temporal segments:
(cents) to tall N components:
"oldt new heuristicic (if and vibrat

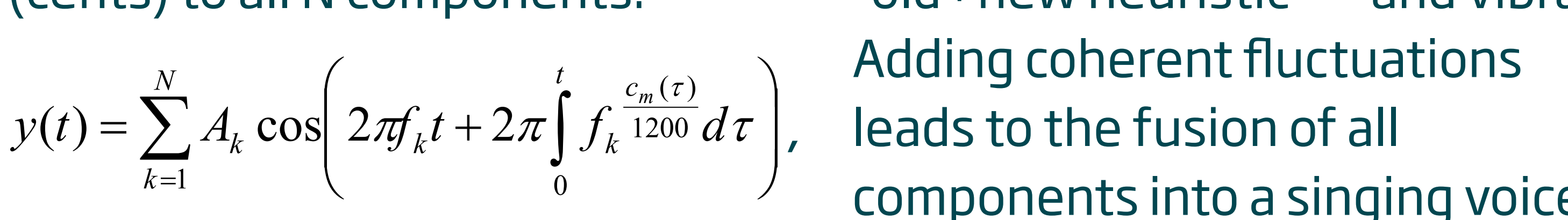

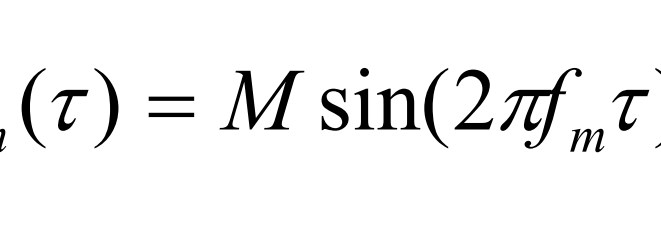

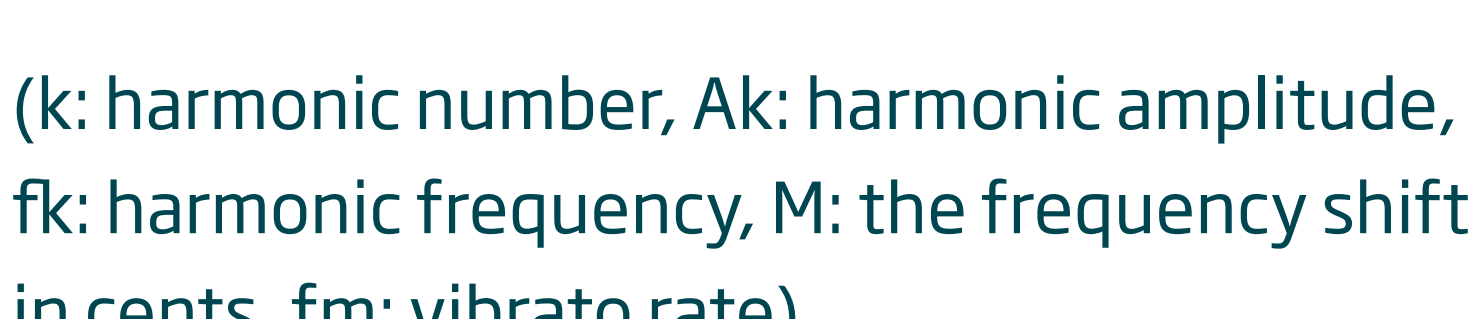

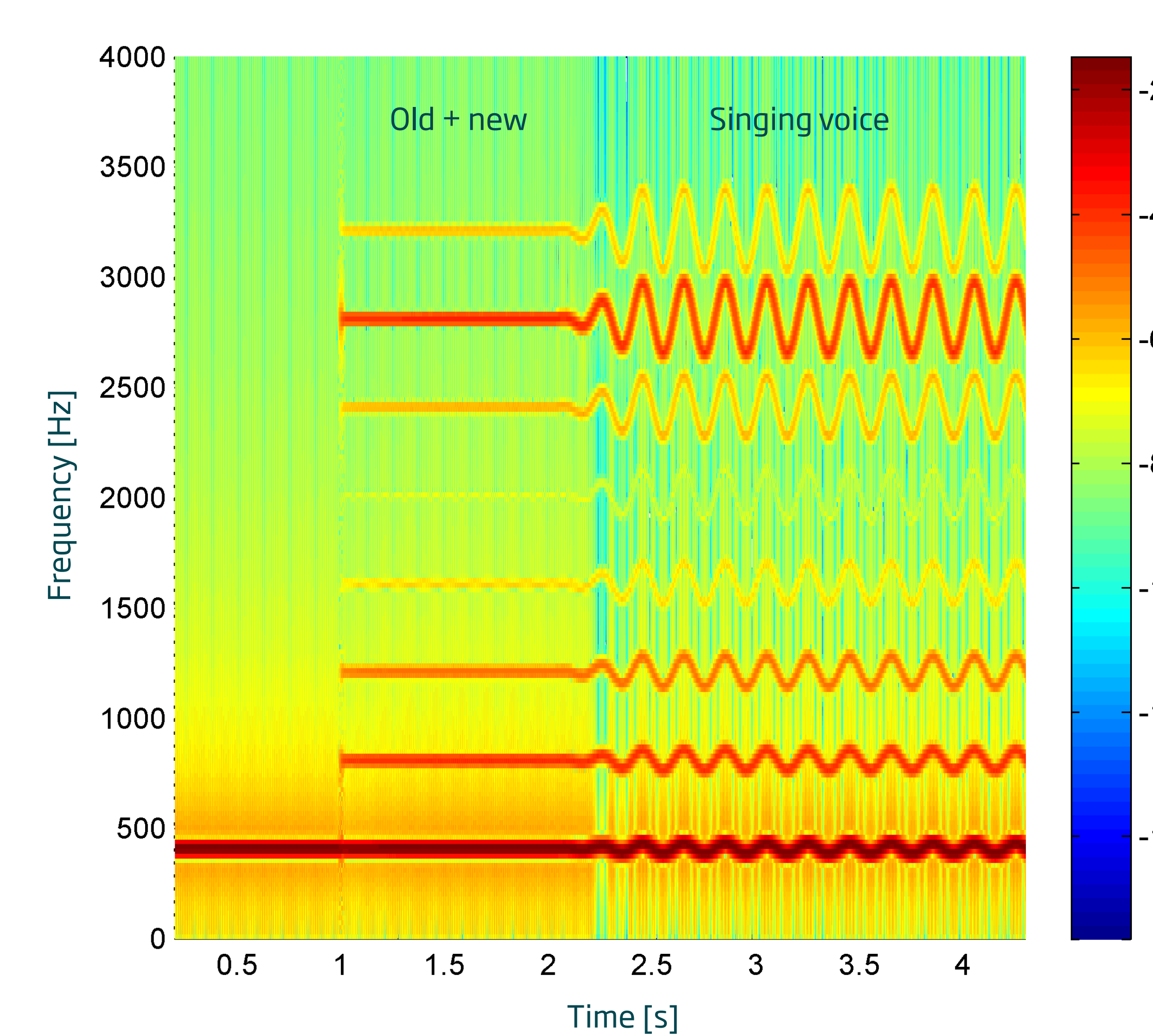

Spectrogram of the basis stimulus, showing its three temporal
segments: 1 fundamental 10 t 400 Hz, z) all eight harmonics of the last segment, the eoubling of the excursion for every harmonic can
be seeen (inear frequency axis). The colors indicate the normalized

- Procedure

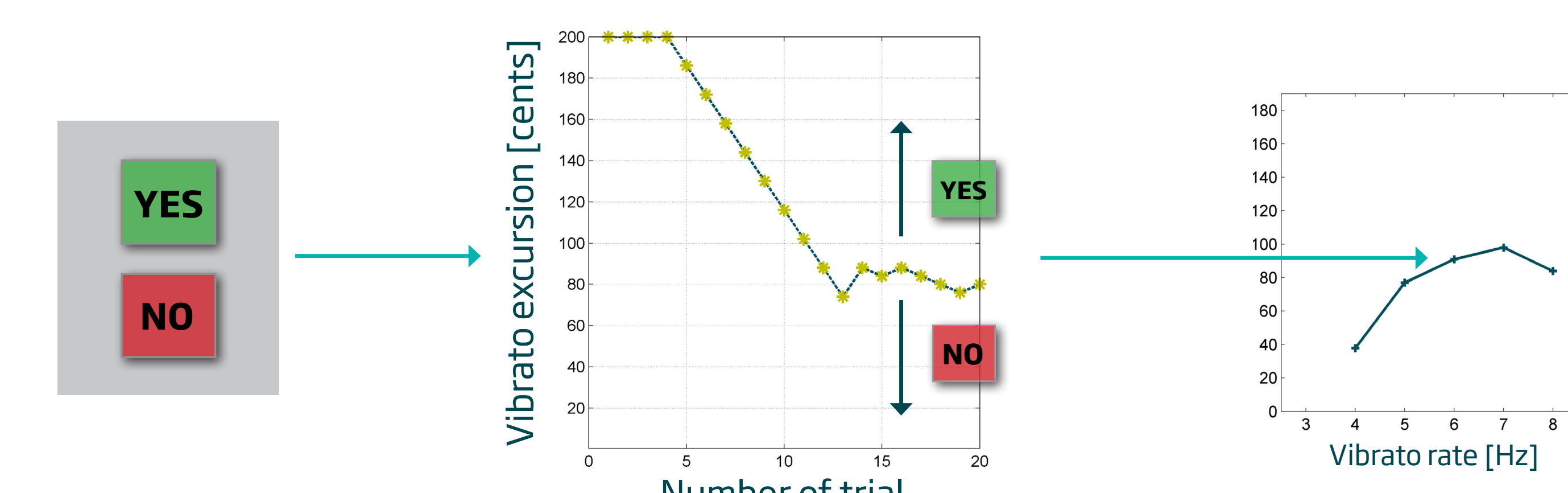

- One-interval, two-alternative, forced-choice "Yes/No" task. - The $50 \%$ point of the psychometric function was derived by means of a
1-up1-down algorithm - Six reference values of vibrato rate: $3,4,5,6,7$, and $8 \mathrm{~Hz}$

- The starting value for the excursion was 200 cents.

- For each presentation of the stimulus, the listeners were requested
to give a"Yres INo" reply to the question can this sound be linked to a to give a "Yes $/ N^{\prime}$ "
singing voice?".

-6 reversals, step sizes of 14 and 4 cents,

$\cdot 3$ repetitions.
- Harmonic complex tone with first
8 Sharmonics of vowimmel / oh/.
Listeners

- All musicians had at least 5 years of mus
instrument or singing on a regular basis.

- Non-musicians had never received any fom not sung or practiced any instrumen

Presentation level

- Stimuli presented diotically at a moderate sound level

- Component levels as in ind with the fundamental component $(400 \mathrm{~Hz})$
set at $70 \mathrm{~dB}$ S SPL.

\section{Results}

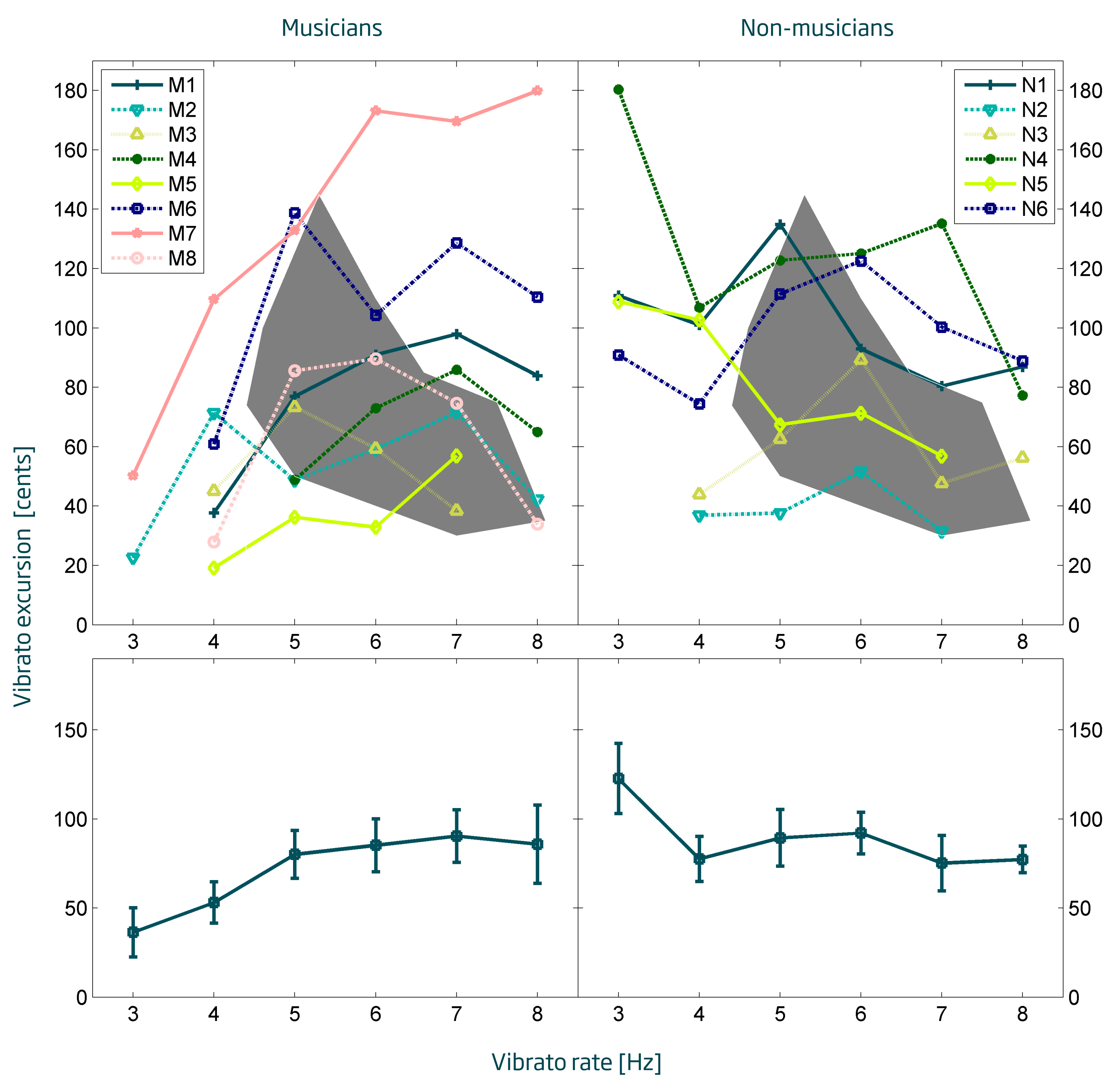

- The thresholds were found to range from 20 to 180 cents for the musi-
cians while this range was from 31 to 180 conts vibrato rates of $4-7$ Hz. However, only 2 musicians and 4 non-musicians

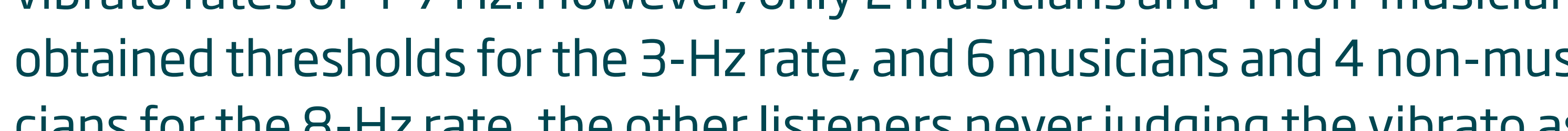
appropriate for these rates.

-A two-way ANOVA showed no S
vibrato rate on the thresholds. - From the individual thresholds it can be seen that eleven out of four-
teen listeners exhnibited a peak in preferreded excursion at the medium
vibrato ates 5 -7 Hzz)

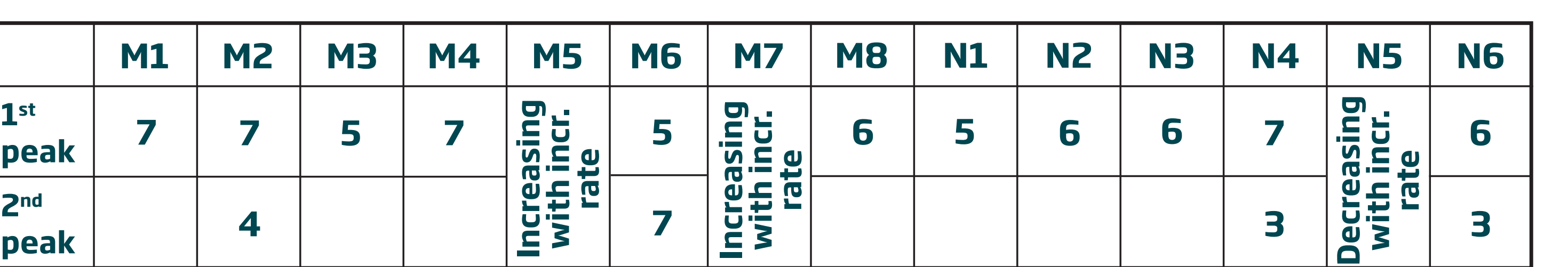

14 $\mathrm{NH}$ listeners (8 musicians [M], 6 non-musicians [N]). M7 and M8 we

\section{Discussion}

Comparison with physical recordings

- The grey area indicates the measuras at $5.5 \mathrm{~Hz}$ between the minimum and maximum excursion produced by

- Thresholds exhibit a peak as a function of rate, but at a rate which did

-There may be a listener-dependent rate range (5-7 $7 \mathrm{~Hz}$ ) within which
larger vibrato excursions are favoured.

Potential explanations for the non-significant effect of vibrato rate

- Thresholds at rates or 3 and $8 \mathrm{~Hz}$ were derived for only yart of the listeners, consistent with the fact that very low and very high values
ofvibrato excursion are shown in the literature not to be preferred by

-Additionally, the individual excursion max
rates for the different listeners $(5-7 \mathrm{~Hz})$.

\section{Conclusion}

- No significant effect of musical experience or rate was found. However

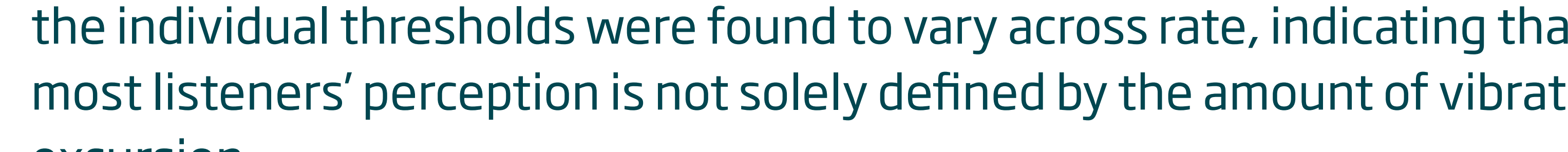
- Large across-subject variability was observed, which may be explained thus the differences in musical preference of the listeners,

- Most of the listeners' thresholds exhibited a peak at medium vibrato
rates These rates did not consistenty corsespond to the 5.5. Hz

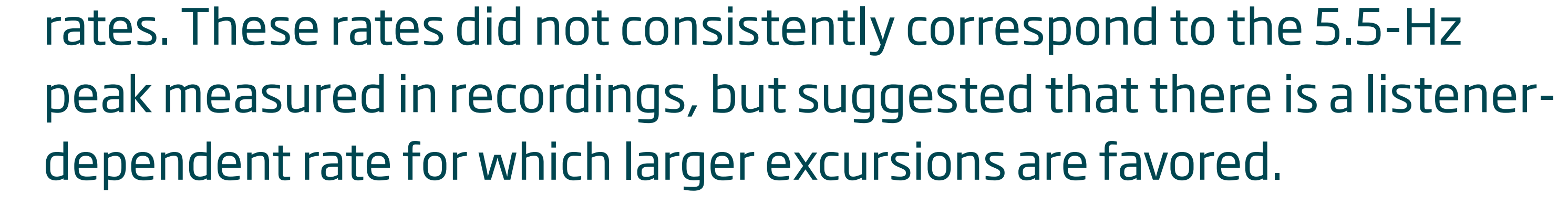

- Further work will investigate modifications on real recordings and also to frequency changes. This would be relevant when studying auditory

$$
\text { (G caHR DTU }
$$

EriksholmResearchCentre 